\title{
Singularly perturbed control systems using non-commutative computer algebra
}

\author{
J. W. Helton ${ }^{1}$, F. Dell Kronewitter ${ }^{1, *, \dagger}$, W. M. McEneaney ${ }^{2}$ and Mark Stankus ${ }^{3}$ \\ ${ }^{1}$ Math Department, University of California, San Diego, 9500 Gilman Dr., San Diego, CA 92093-0112, U.S.A. \\ ${ }^{2}$ Department of Mathematics, North Carolina State University, Raleigh, NC 27695-8205, U.S.A. \\ ${ }^{3}$ Department of Mathematics, California State University, San Luis Obispo, CA 93407, U.S.A.
}

\begin{abstract}
SUMMARY
Most algebraic calculations which one sees in linear systems theory, for example in IEEE TAC, involve block matrices and so are highly non-commutative. Thus conventional commutative computer algebra packages, as in Mathematica and Maple, do not address them. Here we investigate the usefulness of non-commutative computer algebra in a particular area of control theory-singularly perturbed dynamic systems - where working with the non-commutative polynomials involved is especially tedious. Our conclusion is that they have considerable potential for helping practitioners with such computations. Commutative Gröbner basis algorithms are powerful and make up the engines in symbolic algebra packages' Solve commands. Non-commutative Gröbner basis algorithms are more recent, but we shall see that they, together with an algorithm for removing "redundant equations", are useful in manipulating the messy sets of non-commutative polynomial equations which arise in singular perturbation calculations. We use the non-commutative algebra package NCAlgebra and the non-commutative Gröbner basis package NCGB which runs under it on two different problems. We illustrate the method on the classical state feedback optimal control problem, see [1], where we obtain one more (very long) term than was done previously. Then we use it to derive singular perturbation expansions for the relatively new (linear) information state equation. Copyright (C) 2000 John Wiley \& Sons, Ltd.
\end{abstract}

KEY WORDS: non-commutative algebra; computer algebra; dynamic control; singular perturbation; control systems

\section{GEORGE ZAMES}

All of the authors of this paper remember George Zames either through his work or through first-hand experience. George had vast influence on the subject of control and of his accomplishments there are so many accounts that it is pointless to list them here. Not so obvious to an engineering audience is that his invention, $H^{\infty}$ control, led to a fever of activity in

\footnotetext{
* Correspondence to: F. Dell Kronewitter, Math Department, University of California, San Diego, 9500 Gilman Dr., San Diego, CA 92093-0112, U.S.A.

†'E-mail: dell@ieee.org

Contract/grant sponsor: AFOSR

Contract/grant sponsor: NSF
}

Copyright (C) 2000 John Wiley \& Sons, Ltd. 
an area of mathematics called operator theory. Ultimately, this led to a body of beautiful mathematics.

Some personal remarks by one of the authors, Bill Helton, might be appropriate. Always when I think of George I think of how much fun he was. He had a great sense of adventure, curiosity, and even mischievousness. While most people are very cautious in what they say George would speculate about every sort of thing and ask incessant questions out of curiosity and out of an eagerness to get a freewheeling discussion going.

I might add that George in my experience was a "math buff". Besides many illustrations in private conversations of his interest in new mathematical ideas, an example was his regular attendance at the Mathematical Theory of Networks and Systems, to include having the second meeting of the MTNS in 1975 at McGill University, his homebase. This was not the 25th meeting of the MTNS, but was the first tentative feelers (since Norbert Wiener's generation) going out between the engineering community and the operator theory community. Ultimately these interactions led to $H^{\infty}$ control.

The pattern I consistently saw in George was a great sense of adventure in selecting his topic, a relentless focus on the few physical or conceptual issues he thought were central to the topic, and the relentless drive to set these into correspondence with elegant mathematical constructions. I might emphasize this last part, since in modern times young engineers think of George Zames as they would a statue on the engineering school lawn. There is a tendency to think that his achievements came from a very linear process of going from physics to a mathematical treatment. In fact, there was little that was linear about George's thinking and $H^{\infty}$ control at least came from trying relentlessly to set a physical picture and a mathematical picture in correspondence.

\section{INTRODUCTION}

\subsection{Non-commutative algebra on the computer}

While commutative computer algebra has seen heavy development and use, since the MACSYMA project in the 1960s, general non-commutative computer algebra has only recently come to the beginning stages of experimentation; still the field is uncharted and at the stages of high adventure. For perspective, 5 years ago there was little non-commutative algebra software publically available. Unfortunately, to bring non-commutative computer algebra to nearly its potential requires a creation of a small world of algorithms and software.

A crude analogy with the preMATLAB days of engineering comes to mind. Suppose only a few reliable algorithms were known, for example, a (slow) matrix inversion and a (slow) eigensolver is

known; there are no Riccati solvers or other utilities. The field, to get started, faces the task of programming what is known, of doing many experiments to find a collection of successful applications and of developing algorithms to fill major application gaps. That is much like the starting situation with non-commuting computer algebra.

Finally, within the last few years there are pieces of the great software mosaic becoming available. Publicly available programs which are currently maintained and which include a noncommutative Gröbner Basis finder (which in our opinion is like getting to first base) are listed at CAIN, http://www.can.nl. It is not clear which are being supported currently. However, we have had recent contact with the author of OPAL, [2], and Anick, joeb@matematik.su.se. In this paper we use NCGB [3] running under NCAlgebra [4]. 
Our effort, which includes many features, now consists of 1.68 Megabytes of Mathematica code and 2.14 Megabytes of $\mathrm{C}++$ linked to it. Over the last 5 years we have done many experiments on linear systems type of calculations. In this article we report on what appears to be a very successful application of our methods. Much of the work in this paper appears in the Ph.D. Thesis of Dell Kronewitter [5].

\subsection{Singular perturbation vs. computer algebra}

Singular perturbation is a commonly used technique in the analysis of systems whose dynamics consist of two pieces. One piece might be slow, the other fast, or one might be known where the other is somewhat uncertain. Extensive analysis has been done of this type of plant for the LQR and $H^{\infty}$ control problems, for example References $[1,6,7]$.

Typically one has an equation where some coefficients depend on a parameter $1 / \varepsilon$. To solve this equation, one postulates an expansion in $\varepsilon$ for the solutions $x_{\varepsilon}$ to the equation, then

(a) substitutes $x_{\varepsilon}$ into the equation,

(b) sorts the equation according to powers of $\varepsilon$, and

(c) finds simple equations for successive terms in the expansion of $x_{\varepsilon}$.

The sorting in (b) can be tedious and the business of solving (c) can be very involved.

This article concerns methods we are developing for doing these steps automatically. The software runs under NCAlgebra, [4], the most widely distributed Mathematica package for general non-commuting computations. As we shall illustrate, NCAlgebra constructions and commands easily handle steps (a) and (b), thereby producing the (long) list of equations which must be solved. This is straightforward and saves considerable calculation time for those engaged in singular perturbation calculations. Step (c) involves solving complicated systems of equations and this is always tricky business. Thus there is no way of knowing in advance if noncommutative Gröbner basis methods will be effective for (reducing to a simple form) the equations found in large classes of singular perturbation problems. This is the focus of experiments (using the package NCGB which runs under NCAlgebra) we have been conducting and on which we report here.

Most of the paper shows how one can treat the most classic of all singular perturbation problems using computer algebra. Ultimately, we see that Mora's Gröbner basis algorithm together with our algorithm for removing 'redundant equations' is very effective on the equations which result. Indeed our method carries out the expansion one step further than has previously been done, see Section (4.3). Then we sketch another newer $H^{\infty}$ estimation problem called the 'cheap sensor' problem (see Reference [8]). On this our computer techniques proved effective.

\subsection{The idea behind Gröbner computer algebra}

We shall describe some algorithms we use for handling polynomials many of which make use of Gröbner bases.

\subsubsection{Gröbner bases}

We say that a set of equations $\left\{q_{j}=0: 1 \leqslant j \leqslant k\right\}$ eliminates $x_{k}$ if one of the polynomials has the form $q_{j}=x_{k}-r\left(x_{1}, \ldots, x_{k-1}, x_{k+1}, \ldots, x_{n}\right)$ (so that $r$ is a polynomial which does not depend on $x_{k}$ ). 
The non-commutative Gröbner basis algorithm (GBA), due to F. Mora [9], can be used to systematically eliminate variables from a collection (e.g., $\left.\left\{p_{j}\left(x_{1}, \ldots, x_{n}\right)=0: 1 \leqslant j \leqslant k_{1}\right\}\right)$ of polynomial equations so as to put it in triangular form. One specifies an order on the variables $\left(x_{1}<x_{2}<x_{3}<\cdots<x_{n}\right)^{1}$ which corresponds to your priorities in eliminating them. Here a GBA will try hardest to eliminate $x_{n}$ and try the least to eliminate $x_{1}$. The output from it is a list of equations in a 'canonical form' which is triangular: ${ }^{2}$

$$
\begin{aligned}
q_{1}\left(x_{1}\right) & =0 \\
q_{2}\left(x_{1}, x_{2}\right) & =0 \\
q_{3}\left(x_{1}, x_{2}\right) & =0 \\
q_{4}\left(x_{1}, x_{2}, x_{3}\right) & =0 \\
& \vdots \\
q_{k_{2}}\left(x_{1}, \ldots, x_{n}\right) & =0 .
\end{aligned}
$$

Here, the set of solutions to the collection of polynomial equations $\left\{q_{j}=0: 1 \leqslant j \leqslant k_{2}\right\}$ equals the set of solutions to the collection of polynomial equations $\left\{p_{j}=0: 1 \leqslant j \leqslant k_{1}\right\}$. This canonical form greatly simplifies the task of solving the collection of polynomial equations by facilitating back-solving for $x_{j}$ in terms of $x_{1}, \ldots, x_{j-1}$. The effect of the ordering is to specify that variables high in the order will be eliminated while variables low in the order will not be eliminated.

If the variables commute, then the Gröbner basis is always finite and can be generated by Buchberger's algorithm. The Buchberger algorithm always terminates in a finite amount of time. It could terminate in seconds, days, or centuries. In the non-commutative case, which is the subject of this paper, the Gröbner basis is usually infinite and then the GBA fails to halt given finite computational resources. Nevertheless, the solution set of the output of a terminated (say $k$ iteration) GBA, $\left\{q_{j}=0\right\}$, is always equivalent to the solution set of the input, $\left\{p_{i}=0\right\}$, and this partial GBA often proves to be useful in computations as will be shown below. Gröbner basis computer runs can be (notoriously) memory and time consuming. Thus, their effectiveness on any class of problems can only be determined by experiment.

\subsubsection{Removing redundant relations}

There is another part of our algorithm which is important. A Gröbner basis or even a partial Gröbner basis contains many polynomials, a few of which are important since they contain few unknowns. However, they also contain many, many polynomials which are long and uninteresting. Indeed simply producing a Gröbner basis is not that valuable for solving a singular perturbation problem because the gems are buried in the junk.

\footnotetext{
${ }^{1}$ From this ordering on variables (written $<$ ), an order on monomials in those variables is induced which is referred to as non-commutative graded lexicographic order. In this paper we also write $\ll$ to mean a non-commutative pure lexicographic order.

${ }^{2} k_{2}$ may be larger than $n$ (i.e. there need not be $\leqslant n$ equations in the list) and there need not be any equation in just 1 or 2 variables.
} 
We have several algorithms for removing redundant polynomials the specifics of which are described in References [10] and [3]. The one which we have found to be remarkably effective for singular perturbation computations is called RemoveRedundant. Briefly, it records the history of the production of the Gröbner basis as a tree and then throws away all polynomials which correspond to nodes which are not seminal nodes. RemoveRedundant was developed before we undertook this singular perturbation study, so it is gratifying that it works so well on singular perturbation problems.

\subsubsection{NCProcess}

NCProcess can either be viewed as a Mathematica command or a 5 year research project. The general goal of NCProcess is to take a set of non-commutative polynomials and return an equivalent set of non-commutative polynomials which is as 'useful' as possible. The most important tasks NCProcess performs are creating a Gröbner basis, removing redundant polynomials, and categorizing the output.

\subsubsection{Hardware}

Computer computations for Section 4 were performed with NCGB on a Sun Ultra I with one $166 \mathrm{MHz}$ processor and $192 \mathrm{MB}$ of RAM. The computations done in Section 5 were performed with NCGB on a Sun Ultra II with two $166 \mathrm{MHz}$ processors and $1 \mathrm{~Gb}$ of RAM. The Sun Ultra II was a departmental machine and therefore equivalent computations on a dedicated computer might take less than half of the times reported here.

\section{THE STANDARD STATE FEEDBACK SINGULAR PERTURBATION PROBLEM}

The standard singularly perturbed linear-time-invariant model consists of a differential state equation; which depends on some perturbation parameter $\varepsilon$; and an output equation. The general control problem is to design some feedback law which specifies the input as a function of the state so that the controlled system will satisfy some given performance objective.

\subsection{The system}

Here we study the two time scale dynamic system previously analysed in Reference [1]:

$$
\begin{aligned}
{\left[\begin{array}{c}
\frac{\mathrm{d} x}{\mathrm{~d} t} \\
\mathrm{~d} z \\
\mathrm{~d} t
\end{array}\right] } & =\left[\begin{array}{ll}
A_{11} & A_{12} \\
A_{21} & A_{22}
\end{array}\right]\left[\begin{array}{l}
x \\
z
\end{array}\right]+\left[\begin{array}{l}
B_{1} \\
B_{2}
\end{array}\right] u \\
y & =\left[\begin{array}{ll}
M_{1} & M_{2}
\end{array}\right]\left[\begin{array}{l}
x \\
z
\end{array}\right]
\end{aligned}
$$

where $x \in \mathbb{R}^{n}, z \in \mathbb{R}^{m}, u \in \mathbb{R}^{p}$, and $y \in \mathbb{R}^{q}$. Here, $m, n, p$ and $q$ are integers, $A_{11}$ is an $n \times n$ matrix, $A_{12}$ is a $n \times m$ matrix, $A_{21}$ is a $m \times n$ matrix, $A_{22}$ is a $m \times m$ matrix, $B_{1}$ is a $n \times p$ matrix, $B_{2}$ is a $m \times p$ matrix, $M_{11}$ is a $q \times n$ matrix and $M_{22}$ is a $q \times m$ matrix. 


\subsection{The near-optimal $L Q R$ problem}

The infinite-time optimal linear regulator problem is to find a control, $u(t), t \in[0, \infty]$, which minimizes the quadratic cost

$$
J=\int_{0}^{\infty}\left(y^{\mathrm{T}} y+u^{\mathrm{T}} R u\right) \mathrm{d} t
$$

where $R$ is a positive definite weighting matrix. It is well known that the solution to this problem is of the form

$$
u^{*}=-R^{-1} B^{\mathrm{T}} K(\varepsilon)\left[\begin{array}{l}
x \\
z
\end{array}\right]=G(\varepsilon)\left[\begin{array}{l}
x \\
z
\end{array}\right]
$$

where $K(\varepsilon)$ is a solution to the algebraic Riccati equation (ARE)

$$
K A+A^{\mathrm{T}} K-K B R^{-1} B^{\mathrm{T}} K+M^{\mathrm{T}} M=0
$$

with

$$
\begin{gathered}
A=\left[\begin{array}{cc}
A_{11} & A_{12} \\
\frac{A_{21}}{\varepsilon} & \frac{A_{22}}{\varepsilon}
\end{array}\right], \quad B=\left[\begin{array}{l}
B_{1} \\
\frac{B_{2}}{\varepsilon}
\end{array}\right], \quad \text { and } M=\left[\begin{array}{ll}
M_{1} & M_{2}
\end{array}\right] \\
K(\varepsilon)=K_{0}+\varepsilon K_{1}+\varepsilon^{2} K_{2}+\cdots
\end{gathered}
$$

If $K$ is the solution to this optimal state feedback control problem, then it also may be used to express the optimal cost as a function of the initial state of the system as

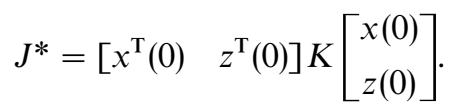

Note that the solution $J^{*}$ as presented involves solving Equation (10) which is a Riccati equation of size $n+m$ by $n+m$. Since the structures present in system (6) are partitioned, it seems likely that we might decompose the problem and significantly reduce the sizes of the matrices while deriving an only slightly sub-optimal controller. Indeed, it is standard to divide the problem of solving the $(n+m)$-dimensional Riccati (10) into solving two smaller decoupled Riccatis, one which is $n$ dimensional, the other which is $m$ dimensional, and then use these solutions to obtain a control law which gives a performance $J$ nearly equal to the optimal performance $J^{*}$. This regulator is known as the near-optimal regulator.

In the next two subsections we review this decomposition of the state space into slow and fast parts. This is mostly a matter of setting our notation, which in fact is the same notation as in Reference [1]. The non-commutative Gröbner computer algebra which is the subject of our investigations is well suited for manipulating polynomials into a triangular or even decoupled form.

\subsection{Decomposing the problem}

Here, we decompose our two time scale system into its fast parts and slow parts. We will assume throughout that $A_{22}$ is invertible. 
3.3.1. The slow system. The dynamics of the slow system can be found by setting $\varepsilon$ to zero in Equation (6) obtaining what is often called the quasi-steady state of the system. This transforms the equation involving $\varepsilon$ in system (6) into an algebraic equation rather than a differential equation,

$$
z_{s}(t)=-A_{22}^{-1}\left(A_{21} x_{\mathrm{s}}(t)+B_{2} u_{\mathrm{s}}(t)\right)
$$

and then substitution of this $z_{s}$ into the top equation in (6) yields

$$
\frac{\mathrm{d} x_{\mathrm{s}}}{\mathrm{d} t}=A_{0} x_{\mathrm{s}}(t)+B_{0} u_{\mathrm{s}}(t), x_{\mathrm{s}}\left(t_{0}\right)=x^{0}
$$

where

$$
A_{0} \triangleq A_{11}-A_{12} A_{22}^{-1} A_{21}, \quad B_{0} \triangleq B_{1}-A_{12} A_{22}^{-1} B_{2}
$$

Here the subscript $s$ indicates that the vectors in Equations (15) and (14) are the slow parts of the vectors in (6).

3.3.2. The fast system. The fast system has dynamics

$$
\frac{\mathrm{d} z_{f}}{\mathrm{~d} t}=A_{22} z_{\mathrm{f}}(t)+B_{2} u_{\mathrm{f}}(t), \quad z_{\mathrm{f}}\left(t_{0}\right)=z^{0}-z_{\mathrm{s}}\left(t_{0}\right)
$$

where

$$
z_{\mathrm{f}}=z-z_{\mathrm{s}} \text { and } \quad u_{\mathrm{f}}=u-u_{\mathrm{s}} .
$$

Here the subscript $f$ indicates that the vectors in Equations (16) and (17) are the fast parts of the vectors in (6).

\subsection{Decomposing the measurement}

We may then also decompose (7) into its slow and fast parts

$$
\begin{aligned}
y & =M_{1} x+M_{2} z \\
z & =M_{1}\left[x_{s}+O(\varepsilon)\right]+M_{2}\left[-A_{22}^{-1}\left(A_{21} x_{s}+B_{2} u_{s}\right)+z_{f}+O(\varepsilon)\right] \\
& =y_{s}(t)+y_{f}(t)+O(\varepsilon)
\end{aligned}
$$

where

$$
y_{s}=M_{0} x_{s}+N_{0} u_{s} \quad \text { and } \quad y_{f}=M_{2} z_{f}
$$

and

$$
M_{0} \triangleq M_{1}-M_{2} A_{22}^{-1} A_{21} \quad \text { and } \quad N_{0} \triangleq-M_{2} A_{22}^{-1} B_{2}
$$




\section{COMPUTER ALGEBRA VS. THE STANDARD SINGULAR PERTURBATION PROBLEM}

The matrix $K(\varepsilon)$ in (12) must be partitioned compatibly with the states $x$ and $z$ and is the limit of the power series which is conventionally written

$$
K_{N}(\varepsilon)=\sum_{i=0}^{N} \varepsilon^{i}\left[\begin{array}{cc}
k_{(1, i)} & \varepsilon k_{(2, i)} \\
\varepsilon k_{(2, i)}^{\mathrm{T}} & \varepsilon k_{(3, i)}
\end{array}\right]
$$

where $k_{(j, i)}$ are appropriately sized matrices (see (6)). We shall use $k_{j i}$ synonymously with $k_{(j, i)}$, since this saves space and actually corresponds to the $\mathrm{T}_{\mathrm{E}} \mathrm{X}$ output of NCAlgebra.

The remainder of this section will be devoted to finding formulas for the $k_{(j, i)}$ for $j \in\{1,2,3\}$ and $i \geqslant 0$.

\subsection{The zero-th order term of the Riccati equation (constant (i.e. $\varepsilon^{0}$ ) coefficients)}

We begin our investigations of the perturbed control problem by searching for the first term of the series (18) consisting of matrices, $k_{(1,0)}, k_{(2,0)}$, and $k_{(3,0)}$. We substitute $K_{0}(\varepsilon)$ into (10) and take only the zeroth-order terms in $\varepsilon$ of the resulting equations. This is problem (b) mentioned in the introduction. In the next section, Section 4.1.1, we will show how this may be done with the assistance of a computer.

This finally brings us to the subject of our investigations, the manipulation of matrix polynomials with computer algebra methods.

4.1.1. Computer algebra finds the basic equations. We begin by using computer algebra to assist in finding the zeroth-order terms in $\varepsilon$ of the equations given in (10).

First, using NCAlgebra, we define the block matrices in (11). The matrix,

$$
\left[\begin{array}{ll}
a & b \\
c & d
\end{array}\right]
$$

is represented in Mathematica by $\{\{a, b\},\{c, d\}\}$. The suffix, $[[j, i]]$, extracts the element in the $j$ th row and the $i$ th column from a given matrix. In NCAlgebra, MatMult performs the matrix multiplication operation, tpMat performs the symbolic transpose operation, and $* *$ indicates non-commutative multiplication.

$$
\begin{aligned}
& A=\{\{\mathrm{A} 11, \mathrm{~A} 12\},\{1 / \operatorname{ep~A21,1/epA2Z~}\}\} \\
& \mathrm{B}=\{\{\mathrm{B} 1\},\{1 / \text { ep B2 }\}\} ; \mathrm{M}=\{\{\mathrm{M} 1, \mathrm{M} 2\}\}
\end{aligned}
$$

We also define a $K_{0}$.

$$
\mathrm{KO}=\{\{\mathrm{klO}, \text { ep kzO }\},\{\text { ep tp[k20], epk30 }\}\}
$$

The following Mathematica function takes as an argument a matrix $K$ and generates the Riccati (10).

$$
\begin{aligned}
\operatorname{Riccati}\left[K_{-}\right]:= & \operatorname{MatMult}[\mathrm{K}, \mathrm{A}]+\operatorname{MatMult}[\mathrm{tpMat}[\mathrm{A}], \mathrm{K}] \\
& -\operatorname{MatMult}[\mathrm{K}, \mathrm{B}, \operatorname{Inv}[\mathrm{R}], \operatorname{tpMat}[\mathrm{B}], \mathrm{K}]+\operatorname{MatMult}[\operatorname{tpMat}[\mathrm{M}], \mathrm{M}]
\end{aligned}
$$


We next use the NCAlgebra command NCTermsOfDegree. ${ }^{3}$ The following Mathematica commands will extract the 0th-order terms in $\varepsilon$, creating the polynomials in (24)-(26):

$$
\begin{array}{ll}
\text { Ep10 = NCTermsOfDegree[Riccati[KO] }[[1,1]], & \{\operatorname{ep}\},\{0\}] \\
\text { Ep20 = NCTermsOfDegree[Riccati[KO] }[[1,2]], & \{\operatorname{ep}\},\{0\}] \\
\text { Ep30 = NCTermsOfDegree [Riccati[KO] }[[2,2]], & \{\operatorname{ep}\},\{0\}]
\end{array}
$$

4.1.1.1. The output. Input (22) creates three polynomials, the third of which is

$$
\mathrm{k} 30 * \text { A22 }+\mathrm{tp}[\mathrm{A} 2 \mathrm{Z}] * * \mathrm{k} 30+\mathrm{tp}[\mathrm{M} 2] * * \mathrm{M} 2-\mathrm{k} 30 * * \mathrm{~B} 2 * * \operatorname{Inv}[\mathrm{R}] * * \operatorname{tp}[\mathrm{B} 2] * * \mathrm{k} 30
$$

When all three are output in $\mathrm{T}_{\mathrm{E}} \mathrm{X}$, which is done easily by NCAlgebra, we get that Riccati $[\mathrm{KO}]=0$ corresponds to the equations

$$
\begin{aligned}
0= & k_{10} A_{11}+A_{11}^{\mathrm{T}} k_{10}+k_{20} A_{21}+A_{21}^{\mathrm{T}} k_{20}^{\mathrm{T}}+M_{1}^{\mathrm{T}} M_{1}-k_{10} B_{1} R^{-1} B_{1}^{\mathrm{T}} k_{10}-k_{20} B_{2} R^{-1} B_{1}^{\mathrm{T}} k_{10} \\
& -k_{10} B_{1} R^{-1} B_{2}^{\mathrm{T}} k_{20}^{\mathrm{T}}+k_{20} B_{2} R^{-1} B_{2}^{\mathrm{T}} k_{20}^{\mathrm{T}} \\
0= & k_{20} A_{22}-k_{20} B_{2} R^{-1} B_{2}^{\mathrm{T}} k_{30}+k_{10} A_{12}+A_{21}^{\mathrm{T}} k_{30}+M_{1}^{\mathrm{T}} M_{2}-k_{10} B_{1} R^{-1} B_{2}^{\mathrm{T}} k_{30} \\
0= & k_{30} A_{22}+A_{22}^{\mathrm{T}} k_{30}+M_{2}^{\mathrm{T}} M_{2}-k_{30} B_{2} R^{-1} B_{2}^{\mathrm{T}} k_{30}
\end{aligned}
$$

4.1.1.2. Simple analysis of the basic equations. Notice that (26), the $T_{E} X$ form of (23), contains only one unknown $k_{30}$ and has the form of a Riccati equation. Thus $k_{30}$ is uniquely determined by this equation if we assume it is the 'stabilizing solution'. That is $A_{22}-B_{2} R^{-1} B_{2}^{\mathrm{T}} k_{30}$ has all eigenvalues in the strict left half plane and is therefore invertible. We have found in our computer experiments that it is best to make heavy invertibility assumptions, especially at the outset. For computer algebra the key property here is invertibility of $A_{22}-B_{2} R^{-1} B_{2}^{\mathrm{T}} k_{30}$.

We may also motivate the invertibility of $A_{22}-B_{2} R^{-1} B_{2}^{\mathrm{T}} k_{30}$ by purely algebraic arguments as follows. Equation (14) contains two unknowns, $k_{10}$ and $k_{20}$, and Equation (25) contains all three unknowns, $k_{10}, k_{20}$, and $k_{30}$. To solve for $k_{20}$ we use Equation (25) and call NCCollect[Ep20, $\mathrm{k20}$ ] to get the following relation:

$$
\begin{aligned}
& \mathrm{k} 20 * *(\mathrm{~A} 22-\mathrm{B} 2 * * \operatorname{Inv}[\mathrm{R}] * * \operatorname{tp}[\mathrm{B} 2] * * \mathrm{k} 30)+\mathrm{tp}[\mathrm{A} 21] * * \mathrm{k} 30+\operatorname{tp}[\mathrm{M} 1] * * \mathrm{M} 2 \\
& -\mathrm{k} 10 * * \mathrm{~B} 1 * * \operatorname{Inv}[\mathrm{R}] * * \operatorname{tp}[\mathrm{B} 2] * * \mathrm{k} 30+\mathrm{k} 10 * * \mathrm{~A} 12
\end{aligned}
$$

Upon examination of (27) it is immediate that we may give $k_{20}$ explicitly in terms of $k_{10}$ and $k_{30}$ by assuming the invertibility of the parenthesized expression in the above relation, $A_{22}-B_{2} R^{-1} B_{2}^{\mathrm{T}} k_{30}$. We have

$$
k_{20}=\left[-k_{10} A_{12}+k_{10} B_{1} R^{-1} B_{2}^{\mathrm{T}} k_{30}-A_{21}^{\mathrm{T}} k_{30}-M_{1}^{\mathrm{T}} M_{2}\right] \cdot\left(A_{22}-B_{2} R^{-1} B_{2}^{\mathrm{T}} k_{30}\right)^{-1} .
$$

We use this expression for $k_{20}$ to change (24) into an equation involving $k_{10}$ and $k_{30}$. The unknown matrices $k_{i 0}$ could then be found by first using (26) to solve for $k_{30}$, then using our transformed (24) to solve for $k_{10}$, and finally using (28) to obtain $k_{20}$. A better situation would be

\footnotetext{
${ }^{3}$ NCTermsOfDegree takes 3 arguments: a (noncommutative) polynomial, a list of variables, and a set of indices. The command returns an expression such that each term is homogeneous with degree given by the indices. For example, the call NCTermsOfDegree[A**B $+\mathrm{B} * * \mathrm{C} * * \mathrm{C}+\mathrm{B} * * \mathrm{~A} * * \mathrm{C}+\mathrm{C} * * \mathrm{D},\{\mathrm{C}\},\{1\}]$ returns $\mathrm{B} * * \mathrm{~A} * * \mathrm{C}+\mathrm{C} * * \mathrm{D}$.
} 
to have some decoupling of the unknown matrices so that certain unknown matrices could be computed concurrently rather than sequentially. Our next objective is to find decoupled equations which determine the unknown matrices.

4.1.2. Heavy analysis of the basic equations. We will show how (24) which involves two unknowns may be replaced by an equation involving only one unknown, $k_{10}$, so that $k_{30}$ and $k_{10}$ may be computed using two independent Riccati equations. We will use the bulk of our Gröbner basis machinery here.

4.1.2.1. Computer algebra jargon. There are several terms we will use which, though simple conceptually, may not be familiar to the control engineer. A product of variables, $x_{1}^{\alpha_{1}} \cdot x_{2}^{\alpha_{2}} \cdots x_{n}^{\alpha_{n}}$ where $\alpha_{k} \in \mathbb{N}$, is called a monomial. A polynomial $f$ is a finite $\mathbb{C}$-linear combination of monomials,

$$
\sum_{j=1}^{m} a_{j} x_{1}^{\alpha_{1}^{j}} \cdot x_{2}^{\alpha_{2}^{j}} \cdots x_{n}^{\alpha_{n}^{j}}
$$

where $a_{j} \in \mathbb{C}$. We call $a_{k}$ the coefficient of the term $a_{k} x_{1}^{\alpha_{1}^{k}} \cdot x_{2}^{\alpha_{2}^{k}} \cdots x_{n}^{\alpha_{n}^{k}}$. A relation is a polynomial which is assumed to be 0 . That is, we may write the equation, $3 x^{2} y z=y z+4 z^{2}$, as a relation, $3 x^{2} y z-y z-4 z^{2}$. Many NCAlgebra functions will accept either relations or equations. We will slip back and forth between the two notations and the meaning should be clear from the context.

4.1.2.2. All algebraic identities which hold. As described in Section 2.3, the GBA takes as input a set of polynomials and an order on the variables involved. It outputs a (generally) more desirable set of equations. It is not necessary to know which polynomials are needed to derive a certain relation. It is only necessary that all needed polynomials are present in the input. For this reason one generally gives as input to the GBA all polynomial relations known to hold. There is no harm in having superfluous (but true) relations in the input. Now we will list the input to our computer algebra program which will generate all polynomial relations known to hold.

First the basic relations we wish to study were produced in the previous section, Ep 10, Ep20 and Ep30; Equations (24)-(26).

In light of the slow system terminology introduced above in Sections 3.3.1 and 3.4 we make the following abbreviations.

$$
\begin{aligned}
& \text { Abbreviations }=\{\mathrm{NO}==-\mathrm{M} 2 * * \operatorname{Inv}[\mathrm{A} 22] * \mathrm{~B} 2, \mathrm{MO}==\mathrm{M} 1-\mathrm{M} 2 * * \operatorname{Inv}[\mathrm{A2Z}] * \text { A21, } \\
& \mathrm{AO}==\mathrm{A} 11-\mathrm{A} 12 * * \operatorname{Inv}[\mathrm{A} 22] * \mathrm{~A} 21, \mathrm{BO}==\mathrm{B} 1-\mathrm{A} 12 * * \operatorname{Inv}[\mathrm{A} 22] * * \mathrm{~B} 2, \\
& \mathrm{RO}==\mathrm{R}+\operatorname{tp}[\mathrm{NO}] * * \mathrm{NO}\}
\end{aligned}
$$

We add the abbreviation $R_{0}$ for convenience as done in Reference [1], although it is not essential. $\operatorname{Inv}[R]$ is the NCAlgebra representation of $R^{-1}$. Since $=$ denotes assignment, Mathematica uses $==$ to denote equality (for equations).

Several of the matrices or matrix polynomials in our problem are assumed to be invertible. It is common to take the matrices, $A_{i i}$, to be of full rank, since otherwise a transformation could be applied to the original system to reduce the size of the state. The matrix $A_{22}-B_{2} R^{-1} B_{2}^{\mathrm{T}} k_{30}$ has already been assumed to be invertible to facilitate the definition of $k_{20}$ in (28). The matrices $R$ and $R_{0}$ are positive definite and so must be invertible. 
We generate the relations which result from these observations with the following command:

$$
\begin{aligned}
\text { Inverses }= & \text { NCMakeRelations }[\{\operatorname{Inv}, \mathrm{R}, \mathrm{RO}, \mathrm{AO}, \mathrm{A} 11, \mathrm{AZZ,} \\
& (\mathrm{A} \text { ¿ }-\mathrm{BZ} * * \operatorname{Inv}[\mathrm{R}] * * \operatorname{tp}[\mathrm{BZ}] * * \mathrm{k} 30)\}]
\end{aligned}
$$

Several of the matrices are known to be self-adjoint, and therefore the following relations must hold:

$$
\begin{aligned}
\text { SelfAdjoints }= & \{\mathrm{k} 10==\mathrm{tp}[\mathrm{k} 10], \mathrm{k} 30=\mathrm{tp}[\mathrm{k} 30], \mathrm{R}==\mathrm{tp}[\mathrm{R}], \mathrm{RO}==\mathrm{tp}[\mathrm{RO}], \\
& \operatorname{Inv}[\mathrm{R}]==\mathrm{tp}[\operatorname{Inv}[\mathrm{R}]], \operatorname{Inv}[\mathrm{RO}]==\mathrm{tp}[\operatorname{Inv}[\mathrm{RO}]]\}
\end{aligned}
$$

We combine all of our relations with

$$
\text { Relations = Union[Ep10, Ep20, Ep30, Abbreviations, SelfAdjoints, Inverses] }
$$

If $p=0$ is a true equation, then $t p[p]==0$ is also. We add these 'transposed' equations

$$
\text { AllRelations = NCAddTranspose[Relations] }
$$

4.1.2.3. Orders. In order to find a polynomial in $k_{10}, A_{0}, B_{0}, M_{0}, N_{0}, R_{0}$, and other variables with a minimal number of occurrences of $k_{20}$, we should create a Gröbner basis for all polynomial relations known to hold under the following order.

$$
N_{0}<M_{0}<R_{0}<A_{0}<B_{0} \ll k_{10} \ll \text { othervariables } \ll k_{20}
$$

Experimenting with other orders is easy, but this one works. The order mentioned in (34) is specified using the NCAlgebra command

$$
\text { NCAutomaticOrder }\{\{\mathrm{NO}, \mathrm{MO}, \mathrm{RO}, \mathrm{AO}, \mathrm{BO}\},\{\mathrm{klO}\},\{\mathrm{Bl}, \mathrm{B} 2, \mathrm{Ml}, \mathrm{M} 2, \mathrm{R}, \mathrm{A} 11, \mathrm{Al} \text {, }
$$

$$
\begin{aligned}
& \mathrm{A} 21, \mathrm{~A} 22, \operatorname{Inv}[\mathrm{A} 22-\mathrm{B} 2 * * \operatorname{Inv}[\mathrm{R}] * * \operatorname{tp}[\mathrm{B} 2] * * \mathrm{k} 30], \mathrm{tp}[\operatorname{Inv}[\mathrm{A} 22-\mathrm{B} 2 * * \\
& \operatorname{Inv}[\mathrm{R}] * * \operatorname{tp}[\mathrm{B} 2] * * \mathrm{k} 30]]\}\{\mathrm{k} 30\},\{\mathrm{k} 20\}\}, \text { AllRelations }]
\end{aligned}
$$

This command scans AllRelations for unassigned letters and places them in the order compatibly with the order given in the first argument.

Finally, the call to make the Gröbner basis is made. This call will create a four iteration partial Gröbner basis from the polynomials included in AllRelations and the output will be stored in the file, 'FindK10'.

$$
\text { NCProcess[AllRelations, 4, "FindK10", SBByCat } \rightarrow \text { False] }
$$

The 'option' SBByCat, which removes large numbers of 'redundant' equations, can be ignored by the reader since in fact we have turned it off to save time.

4.1.3. The output. The output of the command (36) is a set of polynomials which make up the partial Gröbner basis to which RemoveRedundant has been applied created from the polynomials in AllRelations under the order specified in (35). The software we use actually does more than just create a partial Gröbner basis. NCProcess removes redundant relations and categorizes the output depending on how many unknowns lie in each relation. Then it automatically sets them in $\mathrm{T}_{\mathrm{E}} \mathrm{X}, \mathrm{T}_{\mathrm{E}} \mathrm{X}$ 's the file, and opens a window displaying them using 'xdvi'. In this case a category was 
found which consisted of a single relation in the one unknown $k_{10}$ which we will refer to as klOrel. NCProcess automatically performs NCCollect[klOrel, $\mathrm{klO}]$ and displays the expressions with unknown variables $\left\{k_{10}\right\}$ and knowns $\left\{A_{0}, B_{0}, M_{0}, N_{0}, A_{0}^{\mathrm{T}}, B_{0}^{\mathrm{T}}, M_{0}^{\mathrm{T}}, N_{0}^{\mathrm{T}}, R_{0}^{-1}\right\}$

$$
\begin{aligned}
& k_{10}\left(A_{0}-B_{0} R_{0}^{-1} N_{0}^{\mathrm{T}} M_{0}\right)+\left(A_{0}^{\mathrm{T}}-M_{0}^{\mathrm{T}} N_{0} R_{0}^{-1} B_{0}^{\mathrm{T}}\right) k_{10}+M_{0}^{\mathrm{T}} M_{0}-k_{10} B_{0} R_{0}^{-1} B_{0}^{\mathrm{T}} k_{10} \\
& \quad-M_{0}^{\mathrm{T}} N_{0} R_{0}^{-1} N_{0}^{\mathrm{T}} M_{0}
\end{aligned}
$$

This plus Equation (26) gives us desirable decoupled equations for the unknown variables $k_{10}$ and $k_{30}$. It is easy to see from the full output that no equations coupling $k_{10}$ and $k_{30}$ exist. Therefore, we may solve for these unknown matrices concurrently, rather than waiting for the computation of $k_{30}$ to find $k_{10}$ as suggested by the original matrix polynomial form (which is triangular). After solving these two equations for $k_{10}$ and $k_{30}$, we may find $k_{20}$ by substituting $k_{10}$ and $k_{30}$ into (28).

The calculation which computes (37) is no easy feat by hand, as the substitutions and nonstandard notation on p. 116 and 117 of Reference [1] will attest. The answer we found with Gröbner computer algebra is the same as derived there by hand. After the commands were typed into a file, this computation took less than 3 min.

4.1.4. The zeroth-order term of the controller. The optimal controller (9) has first term in $\varepsilon$ equal to

$$
G=-R^{-1}\left[\begin{array}{ll}
B_{1}^{\mathrm{T}} & \frac{B_{2}^{\mathrm{T}}}{\varepsilon}
\end{array}\right]\left[\begin{array}{cc}
k_{(1,0)} & \varepsilon k_{(2,0)} \\
\varepsilon k_{(2,0)}^{\mathrm{T}} & \varepsilon k_{(3,0)}
\end{array}\right] .
$$

and the previous section tells how to compute the $k_{j 0}$.

Note that in many cases it may be advantageous to have an $\varepsilon$ independent controller. Such a goal may be achieved by setting the upper right entry of $K$ to zero. This gives us

$$
\begin{aligned}
G\left[\begin{array}{l}
x \\
z
\end{array}\right] & =\left[\begin{array}{ll}
G_{10} & G_{20}
\end{array}\right]\left[\begin{array}{l}
x \\
z
\end{array}\right] \\
& =-R^{-1}\left(B_{1}^{\mathrm{T}} k_{(1,0)} x+B_{2}^{\mathrm{T}} k_{(2,0)}^{\mathrm{T}} x+B_{2}^{\mathrm{T}} k_{(3,0)} z\right)
\end{aligned}
$$

where $k_{(i, 0)}$ is defined by Equations (37), (28), and (26) for $i$ equal to 1,2, and 3, respectively.

\subsection{The order $\&$ term of the Riccati equation}

In Section 4.1.4, a controller was presented which does not depend on the parameter $\varepsilon$. This is especially appropriate if $\varepsilon$ represents some very small unknown parameter. In fact, there are many circumstances when the parameter $\varepsilon$, while small, is known. In such a case, even though the optimal controller is an infinite power series in $\varepsilon$ one can make an $n$th order approximation to $G(\varepsilon)$ in (9) and arrive at a controller with enhanced performance.

A major obstruction to such an improved approach is the tedious computation required to generate formulas for the coefficients of higher powers of $\varepsilon$. We did not find references where anyone generated formulas for coefficients of $\varepsilon$ higher than 1 . The methods in this paper do, see Section 4.3 . 
As done in Reference [1] we will now obtain decoupled formulas for the matrices $k_{(1,1)}, k_{(2,1)}$, and $k_{(3,1)}$ described in (18). Our approach will require considerably less work than doing it by hand.

Instead of truncating the series (18) to only one term as done in Section 4.1.4 (input (19)) here we define symbolic entries for the second term of $K$ as well.

$\mathrm{K} 1=\{\{\mathrm{k} 10$, ep k20 $\},\{$ ep tp[k20], ep k30\}\} $+\operatorname{ep}\{\{\mathrm{k} 11$, ep k2 1$\},\{$ ep tp[k21], ep k31 $\}\}$

We also append the following abbreviations for the controller discussed above in Section 4.1.4 and defined in Equation (39). These formulas are standard [1]:

$$
\begin{aligned}
\text { Abbreviations }= & \text { Union[Abbreviations, }\{\mathrm{G} 10==-\operatorname{Inv}[\mathrm{R}] * *(\operatorname{tp}[\mathrm{B} 1] * * \mathrm{k} 10 \\
& +\operatorname{tp}[\mathrm{BZ}] * * \operatorname{tp}[\mathrm{k} 20]), \mathrm{G} 20=-\operatorname{Inv}[\mathrm{R}] * * \operatorname{tp}[\mathrm{B} 2] * * \mathrm{k} 30\}] .
\end{aligned}
$$

Since $A_{22}+B_{2} G_{20}=A_{22}-B_{2} R^{-1} B_{2}^{\mathrm{T}} k_{30}$ was previously assumed to be invertible, we also add the invertibility relation,

$$
\text { Inverses }=\text { Union[Inverses, NCMakeRelations[ }\{\text { Inv, }(\mathrm{A} 22+\mathrm{B} 2 * * \mathrm{G} 20)\}]] \text {. }
$$

4.2.1. Extracting the coefficients of $\varepsilon^{1}$. The Riccati expression in $K_{1}$, Riccati[Kl], is an equation with linear and quadratic terms in $\varepsilon$. As done in the last section, the approach here is to equate coefficients of $\varepsilon$. Of course, equating coefficients of $\varepsilon^{2}$ is bogus, since the actual power series (18) would have $k_{(i, 2)}$ which have not been introduced in computer input (40). We can extract the coefficients of $\varepsilon$ in Equation (10) with the following commands:

$$
\text { Epl } 1 \text { = NCTermsOfDegree[Riccati[K1][[1, 1]], }\{\operatorname{ep}\},\{1\}]
$$

creates the following polynomial:

$$
\begin{aligned}
& \text { ep kll**All + ep k2l**A21 + ep tp[Al1]**kll + ep tp[A21]**tp[k21] - }
\end{aligned}
$$

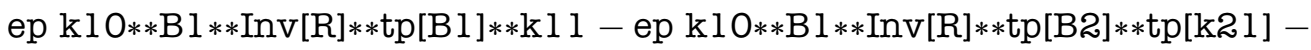

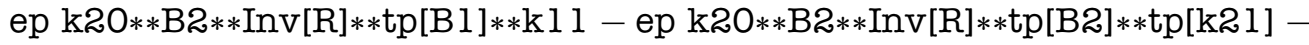

$$
\begin{aligned}
& \text { ep } \mathrm{kl} 1 * * \mathrm{~B} 1 * * \operatorname{Inv}[\mathrm{R}] * * \operatorname{tp}[\mathrm{B} 1] * * \mathrm{k} 10-\operatorname{ep~kl~} 1 * * \mathrm{~B} 1 * * \operatorname{Inv}[\mathrm{R}] * * \operatorname{tp}[\mathrm{B} 2] * * \operatorname{tp}[\mathrm{k} 20]-
\end{aligned}
$$

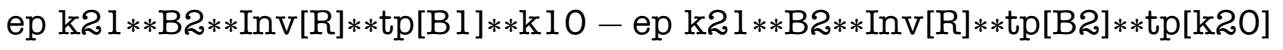

and

$$
\begin{aligned}
& \text { Epz } 1=\text { NCTermsOfDegree[Riccati }[K 1][[1,2]],\{\operatorname{ep}\},\{1\}] \\
& \text { Ep2z = NCTermsOfDegree[Riccati[K1][[2, 2]], }\{\operatorname{ep}\},\{1\}]
\end{aligned}
$$

give similar looking formulas.

4.2.2. Solving for the unknowns. These valid relations can now be added to all relations known to hold, (19), (21), (29), (31), and (30), with the following command. Since the output of the NCTermsOfDegree[ ] command includes the variable ep and we want just the coefficients of ep, 
we set the unwanted variable, ep, to 1 . We do this by appending the Mathematica suffix /.ep $\rightarrow 1$ to expressions involving ep.

$$
\begin{aligned}
& \text { AllRelations = Union[Ep1 1/.ep } \rightarrow \text { 1, Ep21/.ep } \rightarrow 1, \text { Ep31/.ep } \rightarrow 1, \\
& \text { Ep10, Ep20, Ep30, Abbreviations, SelfAdjoints, Inverses] }
\end{aligned}
$$

Considering the analysis done in Section 4.1.1, $k_{(1,0)}, k_{(2,0)}$, and $k_{(3,0)}(\mathrm{k} 10, \mathrm{k} 20, \mathrm{k} 30)$ can be regarded as known and we are now looking at the second term of the series (18), which is made up of these and other knowns and unknowns $\mathrm{kl} 1, \mathrm{k} 21$, and $\mathrm{k} 31$ introduced above in (40). We wish to find simple formulas which determine the unknowns.

With this distinction between known variables and unknown variables, the following order on the variables is appropriate:

$$
\begin{gathered}
N_{0}<M_{0}<R<A_{0}<B_{0}<B_{1}<B_{2}<M_{1}<M_{2}< \\
R_{0}<A_{11}<A_{12}<A_{21}<A_{22}<G_{10}<G_{20}< \\
k_{30}<k_{20}<k_{10} \ll k_{11} \ll \text { other variables }
\end{gathered}
$$

This order is imposed with the command

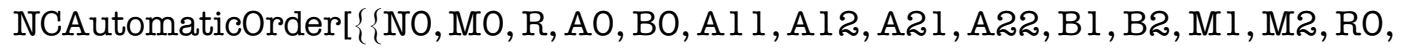

$\mathrm{G} 10, \mathrm{G} 20, \mathrm{k} 30, \mathrm{k} 20, \mathrm{k} 10\},\{\mathrm{k} 11\},\{\mathrm{k} 31\},\{\operatorname{Inv}[\mathrm{A} 22-\mathrm{B} 2 * * \operatorname{Inv}[\mathrm{R}] * * \operatorname{tp}[\mathrm{B} 2] * * \mathrm{k} 30]$,

$$
\operatorname{tp}[\operatorname{Inv}[\mathrm{A} 22-\mathrm{B} 2 * * \operatorname{Inv}[\mathrm{R}] * * \operatorname{tp}[\mathrm{BR}] * * \mathrm{k} 30]]\},\{\mathrm{k} 21\}\}, \text { AllRelations }]
$$

We next call NCProcess with an iteration count of 3 . The computer input is similar to (36). With this input, NCProcess took less than 7 min. The output of this command contains a single relation with 24 terms involving the single unknown matrix $k_{(1,1)}, \mathrm{kl} l \mathrm{rel}$. Collecting around the $\mathrm{kl} l$ with the command NCCollect[kl lrel, $\mathrm{kll}$ l] gives us the following relation:

$$
\begin{aligned}
& -1 k_{11}\left(A_{0}-B_{0} R_{0}^{-1} B_{0}^{\mathrm{T}} k_{01}-B_{0} R_{0}^{-1} N_{0}^{\mathrm{T}} M_{0}\right)+\left(k_{01} B_{0} R_{0}^{-1} B_{0}^{\mathrm{T}}+M_{0}^{\mathrm{T}} N_{0} R_{0}^{-1} B_{0}^{\mathrm{T}}-A_{0}^{\mathrm{T}}\right) k_{11} \\
& +A_{0}^{\mathrm{T}} k_{02} A_{22}^{-1} A_{21}+A_{21}^{\mathrm{T}} A_{22}^{\mathrm{T}-1} k_{02}^{\mathrm{T}} A_{0}-k_{01} B_{0} R_{0}^{-1} B_{0}^{\mathrm{T}} k_{02} A_{22}^{-1} A_{21}-k_{01} B_{0} R_{0}^{-1} B_{2}^{\mathrm{T}} A_{22}^{\mathrm{T}-1} k_{02}^{\mathrm{T}} A_{0} \\
& -A_{0}^{\mathrm{T}} k_{02} A_{22}^{-1} B_{2} R_{0}^{-1} B_{0}^{\mathrm{T}} k_{01}-A_{0}^{\mathrm{T}} k_{02} A_{22}^{-1} B_{2} R_{0}^{-1} N_{0}^{\mathrm{T}} M_{0}-A_{21}^{\mathrm{T}} A_{22}^{\mathrm{T}-1} k_{02}^{\mathrm{T}} B_{0} R_{0}^{-1} B_{0}^{\mathrm{T}} k_{01} \\
& -A_{21}^{\mathrm{T}} A_{22}^{\mathrm{T}-1} k_{02}^{\mathrm{T}} B_{0} R_{0}^{-1} N_{0}^{\mathrm{T}} M_{0}-M_{0}^{\mathrm{T}} N_{0} R_{0}^{-1} B_{0}^{\mathrm{T}} k_{02} A_{22}^{-1} A_{21}-M_{0}^{\mathrm{T}} N_{0} R_{0}^{-1} B_{2}^{\mathrm{T}} A_{22}^{\mathrm{T}-1} k_{02}^{\mathrm{T}} A_{0} \\
& +k_{01} B_{0} R_{0}^{-1} B_{0}^{\mathrm{T}} k_{02} A_{22}^{-1} B_{2} R_{0}^{-1} B_{0}^{\mathrm{T}} k_{01}+k_{01} B_{0} R_{0}^{-1} B_{0}^{\mathrm{T}} k_{02} A_{22}^{-1} B_{2} R_{0}^{-1} N_{0}^{\mathrm{T}} M_{0} \\
& +k_{01} B_{0} R_{0}^{-1} B_{2}^{\mathrm{T}} A_{22}^{\mathrm{T}-1} k_{02}^{\mathrm{T}} B_{0} R_{0}^{-1} B_{0}^{\mathrm{T}} k_{01}+k_{01} B_{0} R_{0}^{-1} B_{2}^{\mathrm{T}} A_{22}^{\mathrm{T}-1} k_{02}^{\mathrm{T}} B_{0} R_{0}^{-1} N_{0}^{\mathrm{T}} M_{0} \\
& +M_{0}^{\mathrm{T}} N_{0} R_{0}^{-1} B_{0}^{\mathrm{T}} k_{02} A_{22}^{-1} B_{2} R_{0}^{-1} B_{0}^{\mathrm{T}} k_{01}+M_{0}^{\mathrm{T}} N_{0} R_{0}^{-1} B_{0}^{\mathrm{T}} k_{02} A_{22}^{-1} B_{2} R_{0}^{-1} N_{0}^{\mathrm{T}} M_{0} \\
& +M_{0}^{\mathrm{T}} N_{0} R_{0}^{-1} B_{2}^{\mathrm{T}} A_{22}^{\mathrm{T}-1} k_{02}^{\mathrm{T}} B_{0} R_{0}^{-1} B_{0}^{\mathrm{T}} k_{01}+M_{0}^{\mathrm{T}} N_{0} R_{0}^{-1} B_{2}^{\mathrm{T}} A_{22}^{\mathrm{T}-1} k_{02}^{\mathrm{T}} B_{0} R_{0}^{-1} N_{0}^{\mathrm{T}} M_{0}
\end{aligned}
$$


The coefficients of $k_{(1,1)}$ in Equation (50), the polynomials in parentheses, suggest that we make the following abbreviation ${ }^{4}$

$$
\mathrm{FO}==\mathrm{AO}-\mathrm{BO} * * \operatorname{Inv}[\mathrm{RO}] * *(\mathrm{tp}[\mathrm{NO}] * * \mathrm{MO}+\mathrm{tp}[\mathrm{BO}] * * \mathrm{kO} 1)
$$

With computer input (51) appended to all relations known to hold, AllRelations, we can put $F_{0}$ low in the order and run NCProcess again, creating a new (partial) Gröbner basis. The output of this command contains the aesthetically pleasing relation defining $k_{(1,1)}$

$$
\begin{aligned}
- & k_{11} F_{0}-1 F_{0}^{\mathrm{T}} k_{11}+A_{21}^{\mathrm{T}}\left(A_{22}+B_{2} G_{20}\right)^{-\mathrm{T}} k_{20}^{\mathrm{T}} F_{0}+F_{0}^{\mathrm{T}} k_{20}\left(A_{22}+B_{2} G_{20}\right)^{-1} A_{21} \\
& +F_{0}^{\mathrm{T}} k_{20}\left(A_{22}+B_{2} G_{20}\right)^{-1} B_{2} G_{10}+G_{10}^{\mathrm{T}} B_{2}^{\mathrm{T}}\left(A_{22}+B_{2} G_{20}\right)^{-\mathrm{T}} k_{20}^{\mathrm{T}} F_{0}
\end{aligned}
$$

This is a simple Lyapunov equation whose solution, $k_{(1,1)}$, is unique as long as $\mathrm{FO}$, is Hurwitz. We will therefore regard $k_{(1,1)}$ as known from this point forward.

Similar to the zeroth-order case the equation defining $k_{(3,1)}$ is an immediate consequence of (53) and takes the collected form

$$
\begin{aligned}
& k_{31}\left(A_{22}-B_{2} R^{-1} B_{2}^{\mathrm{T}} k_{30}\right)+\left(A_{22}^{\mathrm{T}}-k_{30} B_{2} R^{-1} B_{2}^{\mathrm{T}}\right) k_{31}+A_{12}^{\mathrm{T}} k_{20}+k_{02}^{\mathrm{T}} A_{12} \\
& \quad-k_{30} B_{2} R^{-1} B_{1}^{\mathrm{T}} k_{20}-k_{20}^{\mathrm{T}} B_{1} R^{-1} B_{2}^{\mathrm{T}} k_{30}
\end{aligned}
$$

Also similar to the zero-th order case we have an explicit formula for $k_{(2,1)}$ in terms of $k_{(1,1)}$ and $k_{(3,1)}$. The following relatively simple formula was also in the output of the NCProcess command which generated (52).

$$
\begin{aligned}
k_{21} \rightarrow & -1 k_{11} A_{12} A_{22}^{-1}-A_{11}^{\mathrm{T}} k_{20}\left(A_{22}+B_{2} G_{20}\right)^{-1}-A_{21}^{\mathrm{T}} k_{31}\left(A_{22}+B_{2} G_{20}\right)^{-1} \\
& -G_{10}^{\mathrm{T}} B_{1}^{\mathrm{T}} k_{20}\left(A_{22}+B_{2} G_{20}\right)^{-1}-G_{10}^{\mathrm{T}} B_{2}^{\mathrm{T}} k_{31}\left(A_{22}+B_{2} G_{20}\right)^{-1} \\
& +k_{11} B_{0} R_{0}^{-1}\left(N_{0}^{\mathrm{T}} M_{2} A_{22}^{-1}-B_{2}^{\mathrm{T}} A_{22}^{\mathrm{T}-1} k_{30}\right)
\end{aligned}
$$

Here, and in the rest of the paper, an arrow can be interpreted to mean equal sign. Expressions equivalent to (52)-(54) can be found in Reference [1].

Note that a similar procedure could be done as described in Section 4.1.4 to derive an order $\varepsilon$ controller.

\subsection{The order $\varepsilon^{2}$ term of the Riccati equation}

At this point the tale is growing long and the weary reader can most likely guess what will be done in this section from the title. For the sake of presenting a formula which has not appeared before we create a three-term approximation to $K(\varepsilon)$,

$$
\begin{aligned}
& \mathrm{K} 2=\{\{\mathrm{k} 10, \text { ep k20 }\},\{\text { ep tp[k20], ep k30 }\}\} \\
& +\operatorname{ep}\{\{\mathrm{k} 11, \text { ep k21\}, } \operatorname{ep} \mathrm{tp}[\mathrm{k} 21], \operatorname{ep~k} 31\}\} \\
& \text { + ep } 2\{\{k 21, \text { ep k2え\}, \{ep tp[k2Z], ep k32\}\}, }
\end{aligned}
$$

\footnotetext{
${ }^{4}$ More analytic methods can be used [1] to show that this expression (51) is of the form $A_{0}+B_{0} G_{0}$ where $G_{0}$ is the optimal control for the slow part of the LQR optimal problem (15). The focus here is on the computer algebra and the expression, $F_{0}$, is discovered purely algebraically.
} 
For this problem a three iteration partial Gröbner basis was created and we arrived at formulas defining $k_{(1,2)}, k_{(2,2)}$, and $k_{(3,2)}$. Even without running NCProcess one sees that $k_{(3,2)}$ satisfies a Riccati equation. This is analogous to the lower order cases.

We found one equation which expresses $k_{(2,2)}$ in terms of $k_{(1,2)}$ and $k_{(3,2)}$. We found a Lyapunov equation in the unknown $k_{(1,2)}$ consisting of 150 lines in Mathematica notation. There were also several equations in the unknowns $k_{(1,2)}$ and $k_{(3,2)}$. In analogy with the lower order cases we expect that these 'coupled' equations are redundant and provided no additional information or contraints. Our algebraic software in principle if given enough time can determine this but these algorithms are more computer intensive and did not finish when run on this problem.

We used a version of NCProcess which was specialized to display only equations involving the unknowns; $k_{(1,2)}$ and $k_{(2,2)}$. This substantially speeds up run times. Still, our rather formidable conclusion took $21.5 \mathrm{~min}$. The formulas can be found at

$$
\text { http://math.ucsd.edu/ ncalg/SingularPerturbation. }
$$

It is gratifying that our Gröbner equation processing techniques prevailed on such a large problem. It leads us to think that many singular perturbation problems are well within the scope of our computer algebra techniques.

\section{PERTURBING SINGULAR SOLUTIONS OF THE INFORMATION STATE EQUATION}

We would also like to mention that the techniques illustrated on the previous problem apply to other problems. In particular, we mention a singular perturbation analysis of an important entity in the output feedback $H^{\infty}$ control problem, the information state. It corresponds not to fast and slow time scales but to sensors becoming increasingly accurate, for details see Reference [8].

\subsection{The general problem}

Consider the system

$$
\begin{aligned}
& \frac{\mathrm{d} x}{\mathrm{~d} t}=A(x)+B(x) v \\
& \text { out }=C(x)+D(x)
\end{aligned}
$$

An equation useful in estimation associated to this (details in Reference [8]) is the information state equation (ISE)

$$
\begin{aligned}
-\frac{\mathrm{d} p}{\mathrm{~d} t}= & (A(x)+B(x) \cdot v(t))^{\mathrm{T}} \nabla_{x} p_{t}(x)-\left(\nabla_{x} p_{t}(x)\right)^{\mathrm{T}} Q(x) \nabla_{x} p_{t}(x)-[C(x)-D v(t)]^{\mathrm{T}} J[C(x)-D v(t)] \\
& +\frac{1}{\varepsilon^{2}}(L v(t)-x)^{\mathrm{T}} R(L v(t)-x)
\end{aligned}
$$

where $J$ is self-adjoint. 


\subsection{The linear case}

Assuming that the associated vector field is linear and $\varepsilon$ is fixed, it is known that a solution exists of the form

$$
p_{t}(x)=\frac{1}{2}\left(x-x_{\varepsilon}\right)^{\mathrm{T}} P_{\varepsilon}\left(x-x_{\varepsilon}\right)+\phi^{\varepsilon}(t)
$$

where $P_{\varepsilon}$ is a symmetric matrix which does not depend on $t$, but $x$ and $x_{\varepsilon}$ do depend on $t$. Then

$$
\nabla_{x} p=P_{\varepsilon}\left(x-x_{\varepsilon}\right) .
$$

Noting that $(A x+B v(t))^{\mathrm{T}} \nabla p$ is scalar, we can symmetrize Equation (58), the (ISE), and arrive at

$$
\begin{aligned}
-\frac{\mathrm{d} p}{\mathrm{~d} t}=[ & A x+B v(t)]^{\mathrm{T}} P_{\varepsilon}\left(x-x_{\varepsilon}\right)+\left(x-x_{\varepsilon}\right)^{\mathrm{T}} P_{\varepsilon}[A x+B v(t)] \\
& -\left(x-x_{\varepsilon}\right)^{\mathrm{T}} P_{\varepsilon} Q P_{\varepsilon}\left(x-x_{\varepsilon}\right)-[C x-D v(t)]^{\mathrm{T}} J[C x-D v(t)] \\
& +\frac{1}{\varepsilon^{2}}(L v(t)-x)^{\mathrm{T}} R(L v(t)-x)
\end{aligned}
$$

where $R$ is positive semi-definite.

In seeking an asymptotic expansion one can try various possible forms for a solution. We have found our computer algebra methods very effective in going from a postulated form of an expansion to explicit formulas for their coefficients. Let us illustrate this with the following postulates for $P_{\varepsilon}$ and $x_{\varepsilon} . P_{\varepsilon}$ is a function of $\varepsilon$ which has a series form

$$
P_{\varepsilon}=\frac{1}{\varepsilon} P_{-1}+P_{0}+\varepsilon P_{1}+\varepsilon^{2} P_{2}+\cdots
$$

and we expand $x_{\varepsilon}$ as

$$
x_{\varepsilon}(t)=x_{\varepsilon, 0}(t)+\varepsilon x_{\varepsilon, 1}(t)+\varepsilon^{2} x_{\varepsilon, 2}(t)+\cdots
$$

The treatment of $\phi^{\varepsilon}$ is less critical as we shall see.

\subsection{Finding the expansions of unknowns using computer algebra}

We now apply NCAlgebra methods very similar to the ones demonstrated in Section 4 to the ISE singular perturbation problem. We do not give as much detail since now the idea should be clear. However, we state our expansions precisely, since this must be done to set notation.

5.3.1. Setting the expansions of unknowns. We will begin by creating a symbolic entry for $P_{\varepsilon}$. This is done with the following computer input:

$$
\mathrm{Pe}=(1 / \mathrm{ep}) \mathrm{Pm} 1+\mathrm{PO}+\text { ep Pl }+ \text { ep } 2 \mathrm{P} 2+\text { ep } 3 \mathrm{P} 3
$$

where ep, the symbolic entry for $\varepsilon$, is declared to be commutative and Pm 1, PO, P1, P2, and P3, symbolic entries for $P_{-1}, P_{0}, P_{1}, P_{2}$, and $P_{3}$, respectively, are declared to be non-commutative.

We do not need a formula for $p_{t}$ itself, since what we use is GP $=\nabla_{x} p_{t}(x)$. It is

$$
\mathrm{GP}=\mathrm{Pe} * *(\mathrm{x}-\mathrm{xe}) .
$$


The $x_{\varepsilon}$ described in (62) is defined next

$$
\mathrm{xe}=\mathrm{xeO}+\mathrm{ep} \mathrm{xel}+\mathrm{ep} \hat{\mathrm{z}} \mathrm{xe} 2+\mathrm{ep} \mathbf{3} \mathrm{xe} 3
$$

We also need the derivative $\mathrm{d} x_{\varepsilon} / \mathrm{d} t$ and it has the expansion

$$
d x e=d x e 0+\text { ep dxel }+ \text { ep } 2 d x e z+\text { ep } 3 \text { dxe } 3
$$

Since $\phi^{\varepsilon}(t)$ introduced in Equation (59) does not depend on $x$, its derivative in $t$ appears and that is on the left side of (60). This means that whatever we find as an approximation to $P_{\varepsilon}$ and $x_{\varepsilon}$ can be put on the right-hand side of the equation

$$
-\frac{\mathrm{d} \phi^{\varepsilon}(t)}{\mathrm{d} t}=-(B v)^{\mathrm{T}} P_{\varepsilon} x_{\varepsilon}-x_{\varepsilon}^{\mathrm{T}} P_{\varepsilon} B v+x_{\varepsilon}^{\mathrm{T}} P_{\varepsilon} Q P_{\varepsilon} x_{\varepsilon}+(D v)^{\mathrm{T}} J D v+\frac{1}{\varepsilon^{2}}(L v)^{\mathrm{T}} R L v
$$

which can be integrated to produce $\phi^{\varepsilon}(t)$. Also we can expand $\mathrm{d} \phi^{\varepsilon}(t) / \mathrm{d} t$ out a few terms in $\varepsilon$ and obtain formulas for these terms in the series.

5.3.2. The equations. The ISE (58) is implemented next, and the command NCTermsOfDegree is used to sort this by powers of $\varepsilon$ and $x$. This produced an equation from each coefficient of $1 / \varepsilon^{2}$, $1 / \varepsilon, \varepsilon^{0}$, and $x, x^{\mathrm{T}}$ and $x$ and $x^{\mathrm{T}}$, to produce a set SE of equations. We did not include terms without $x$ and so no $\phi$ 's will appear in SE. Details are strictly analogous to what we just did in Section 4 so they will be omitted.

We will assume the matrix $A$ is invertible, by defining its inverse, $\operatorname{Inv}[\mathrm{A}]$ :

$$
\text { inverses }=\{\operatorname{Inv}[\mathrm{A}] * * \mathrm{~A}-1==0, \mathrm{~A} * * \operatorname{Inv}[\mathrm{A}]-1==0\} .
$$

Many of our matrices are known to be self adjoint, so the equations establishing this are also included with SE and inverses to obtain the full set of equations we put into our Gröbner basis algorithm.

5.3.3. Applying the Gröbner basis algorithm. We need to select a monomial order. $A, B, C, D, J, Q, R, v$, and $L$ are known and we certainly do not wish to solve for them, so we set them at the bottom of the order. Since the $P_{\varepsilon}$ and $x_{\varepsilon}$ are unknown and must be solved for, we set the symbolic unknowns in their expansion above the knowns in the monomial order. The order we choose on the unknowns puts $P_{\varepsilon}$ at the bottom of the unknowns, but still above the knowns which means that once they are solved for they can be used in in formulas for $x_{\varepsilon}$. To implement this and automatically impose a corresponding order on transposes and inverses of variables we use the command NCAutomaticOrder and produce the following order:

$$
\begin{aligned}
A & <A^{-1}<A^{\mathrm{T}}<B<B^{\mathrm{T}}<Q<J<C<C^{\mathrm{T}}<R<D<D^{\mathrm{T}}<v<v^{\mathrm{T}}<L<L^{\mathrm{T}} \\
& \ll P m_{1}<P_{0}<P_{1}<P_{2}<P_{3} \ll x e_{0}<x e_{0}^{\mathrm{T}} \ll x e_{1}<x e_{1}^{\mathrm{T}} \ll x e_{2}<x e_{2}^{\mathrm{T}} \ll x e_{3}<x e_{3}^{\mathrm{T}} \\
& \ll \mathrm{d} x e_{0}<\mathrm{d} x e_{0}^{\mathrm{T}} \ll \mathrm{d} x e_{1}<\mathrm{d} x e_{1}^{\mathrm{T}} \ll \mathrm{d} x e_{2}<\mathrm{d} x e_{2}^{\mathrm{T}} \ll \mathrm{d} x e_{3}<\mathrm{d} x e_{3}^{\mathrm{T}} .
\end{aligned}
$$

Finally a partial Gröbner basis is created and 'redundant equations' are removed using the NCProcess[ ] command

NCProcess[AllRelations, 3, “Answer"] 


\subsection{The answer}

The output of this command includes the following relations which will give us formulas and differential equations which have been derived purely algebraically. The computations took $3 \mathrm{~min}$ and $7 \mathrm{~s}$.

5.4.1. Relations defining $P_{\varepsilon}$. The relations involving $P_{\varepsilon}$ in the output of NCProcess are exactly the following.

The expressions with unknown variables $\left\{P m_{1}\right\}$ and knowns $\{Q, R\}$

$$
P m_{1} Q P m_{1} \rightarrow R
$$

The expressions with unknown variables $\left\{P_{0}, P m_{1}\right\}$ and knowns $\left\{A, Q, A^{\mathrm{T}}\right\}$

$$
P_{0} Q P m_{1} \rightarrow 1 / 2 P m_{1} A+1 / 2 A^{\mathrm{T}} P m_{1}-P m_{1} Q P_{0}
$$

The expressions with unknown variables $\left\{P_{1}, P_{0}, P m_{1}\right\}$ and knowns $\left\{A, C, J, Q, A^{\mathrm{T}}, C^{\mathrm{T}}\right\}$

$$
P_{1} Q P m_{1} \rightarrow 1 / 2 P_{0} A+1 / 2 A^{\mathrm{T}} P_{0}-P_{0} Q P_{0}-P m_{1} Q P_{1}-C^{\mathrm{T}} J C
$$

The expressions with unknown variables $\left\{P_{2}, P_{1}, P_{0}, P m_{1}\right\}$ and knowns $\left\{A, Q, A^{\mathrm{T}}\right\}$

$$
P_{2} Q P m_{1} \rightarrow 1 / 2 P_{1} A+1 / 2 A^{\mathrm{T}} P_{1}-P_{0} Q P_{1}-P_{1} Q P_{0}-P m_{1} Q P_{2}
$$

Indeed this TeX display is exactly what one sees on the screen. In view of the above equations we have the following recursive formula for the matrices $P_{i}$. We begin with the defining relation for $P_{-1}$.

$$
P_{-1} Q P_{-1}=R
$$

Then we must also have that $P_{0}$ satisfies the algebraic Lyapunov equation

$$
P_{-1} A+A^{\mathrm{T}} P_{-1}=2\left(\left[\begin{array}{ll}
P_{0} Q & P_{-1} Q
\end{array}\right]\left[\begin{array}{c}
P_{-1} \\
P_{0}
\end{array}\right]\right)
$$

and $P_{1}$ satisfies

$$
P_{0} A+A^{\mathrm{T}} P_{0}=2\left(\left[\begin{array}{lll}
P_{1} Q & P_{0} Q & P_{-1} Q
\end{array}\right]\left[\begin{array}{c}
P_{-1} \\
P_{0} \\
P_{1}
\end{array}\right]+C^{\mathrm{T}} J C\right)
$$

Inspection strongly suggests that the coefficient matrices of higher powers of $\varepsilon$ are given by the recursive formula

which alternatively may be written as

$$
P_{i-1} A+A^{\mathrm{T}} P_{i-1}=2\left(\left[\begin{array}{llll}
P_{-1} Q & P_{0} Q & \cdots & P_{i} Q
\end{array}\right]\left[\begin{array}{c}
P_{i} \\
\vdots \\
P_{0} \\
P_{-1}
\end{array}\right]\right)
$$

$P_{i} Q P_{-1}+P_{-1} Q P_{i}=1 / 2\left(P_{i-1} A+A^{\mathrm{T}} P_{i-1}\right)-P_{i-1} Q P_{0}-P_{i-2} Q P_{1}-\cdots-P_{1} Q P_{i-2}-P_{0} Q P_{i-1}$ 
In many cases we will have $Q=B B^{\mathrm{T}}$ where $B$ is an input matrix corresponding to a dynamical system. Since we expect $B$ to be a tall, skinny matrix we also expect $B B^{\mathrm{T}} P_{-1}$ to have a significant number of eigenvalues equal to zero and hence have a high degree of non uniqueness in the $P_{i}$ solving Equation (77).

5.4.2. Relations defining $x_{\varepsilon}(t)$. The entire output of NCProcess which involves $x_{\varepsilon}$ is exactly the following plus their transposes.

The expressions with unknown variables $\left\{x e_{0}\right\}$ and knowns $\{L, R, v\}$

$$
R\left(x e_{0}-L v\right)==0
$$

The expressions with unknown variables $\left\{\mathrm{d} x e_{0}, x e_{1}, x e_{0}, P m_{1}\right\}$ and knowns $\{A, B, R, v\}$

$$
P m_{1} \mathrm{~d} x e_{0} \rightarrow 2 R x e_{1}+P m_{1} A x e_{0}+P m_{1} B v
$$

The expressions with unknown variables $\left\{\mathrm{d} x e_{1}, \mathrm{~d} x e_{0}, x e_{2}, x e_{1}, x e_{0}, P_{0}, P m_{1}\right\}$ and knowns $\left\{A, B, C, D, J, R, v, C^{\mathrm{T}}\right\}$

$$
\begin{aligned}
P m_{1} \mathrm{~d} x e_{1} \rightarrow & -1 P_{0} \mathrm{~d} x e_{0}+2 R x e_{2}+P_{0} A x e_{0}+P_{0} B v+P m_{1} A x e_{1} \\
& -2 C^{\mathrm{T}} J C x e_{0}+2 C^{\mathrm{T}} J D v
\end{aligned}
$$

The expressions with unknown variables $\left\{\mathrm{d} x e_{2}, \mathrm{~d} x e_{1}, \mathrm{~d} x e_{0}, x e_{3}, x e_{2}, x e_{1}, x e_{0}, P_{1}, P P_{0}, P m_{1}\right\}$ and knowns $\left\{A, B, C, J, R, v, C^{\mathrm{T}}\right\}$

$$
\begin{aligned}
P m_{1} \mathrm{~d} x e_{2} \rightarrow & -1 P_{0} \mathrm{~d} x e_{1}-P_{1} \mathrm{~d} x e_{0}+2 R x e_{3}+P_{0} A x e_{1}+P_{1} A x e_{0}+P_{1} B v \\
& +P m_{1} A x e_{2}-2 C^{\mathrm{T}} J C x e_{1}
\end{aligned}
$$

These relations may be written quite succinctly in the following way.

We have the following relations governing the behaviour of the terms of the expansion of $x_{\varepsilon}$ introduced in Equation (62).

$$
x_{\varepsilon, 0}=L v \text { on the subspace orthogonal to the kernel of } R
$$

or in other words

$$
R\left(x_{\varepsilon, 0}-L v\right)=0 .
$$

The terms $x_{\varepsilon, 0}, x_{\varepsilon, 1}$, and $x_{\varepsilon, 2}$ are governed by the pair of differential equations

$$
\begin{gathered}
P_{-1} \frac{\mathrm{d} x_{\varepsilon, 0}}{\mathrm{~d} t}=P_{-1} A x_{\varepsilon, 0}+2 R x_{\varepsilon, 1} \text { and } \\
\mathrm{P}_{-1} \frac{\mathrm{d} x_{\varepsilon, 1}}{\mathrm{~d} t}+P_{0} \frac{\mathrm{d} x_{\varepsilon, 0}}{\mathrm{~d} t}=\left[\begin{array}{ll}
P_{-1} A & P_{0} A
\end{array}\right]\left[\begin{array}{c}
x_{\varepsilon, 1} \\
x_{\varepsilon, 0}
\end{array}\right]+2 R x_{\varepsilon, 2}-2 C^{\mathrm{T}} J C x_{\varepsilon, 0}+2\left(C^{\mathrm{T}} J D+P_{0} B\right) v
\end{gathered}
$$


and then the higher terms, $x_{\varepsilon, k}$ for $k \geqslant 2$, satisfy

$$
\begin{aligned}
& {\left[\begin{array}{llll}
P_{-1} & P_{0} & \ldots & P_{k-1}
\end{array}\right]\left[\begin{array}{c}
\frac{\mathrm{d} x_{\varepsilon, k}}{\mathrm{~d} t} \\
\vdots \\
\frac{\mathrm{d} x_{\varepsilon, 1}}{\mathrm{~d} t} \\
\frac{\mathrm{d} x_{\varepsilon, 0}}{\mathrm{~d} t}
\end{array}\right]=\left[\begin{array}{llll}
P_{-1} A & P_{0} A & \ldots & P_{k-1} A
\end{array}\right]\left[\begin{array}{c}
x_{\varepsilon, k} \\
\vdots \\
x_{\varepsilon, 1} \\
x_{\varepsilon, 0}
\end{array}\right]} \\
& -2 C^{\mathrm{T}} J C x_{\varepsilon, k-1}+2 R x_{\varepsilon, k+1}+P_{k-1} B v
\end{aligned}
$$

5.4.3 Relations defining $\phi^{\varepsilon}(t)$. There are two ways to proceed for computing $\phi^{\varepsilon}(t)$. An approximation to $\phi^{\varepsilon}(t)$ may be computed using the formula (67) along with the expansions just obtained.

The longer method which we do not reproduce here is to give full expansions for $\phi^{\varepsilon}(t)$ in terms of $\phi_{l}^{\varepsilon}(t)$ where $l \geqslant-2$. We did this with our computer and found rather long formulas for the first few terms. This was easy, but there is no point in listing them here.

\section{REFERENCES}

1. Kokotovic PV, Khalil HK, O'Reilly J. Singular Perturbation Methods in Control: Analysis and Design. Academic Press: New York, 1986.

2. Keller B, Green E. The OPAL System. Available from http://hal.cs.vt.edu/opal, 1998.

3. Helton JW, Stankus M. NCGB: Noncommutative Groebner Bases. Available from http://math.ucsd.edu/ ncalg, 1997.

4. Helton JW, Miller RL, Stankus M. NCAlgebra: A Mathematica Package for doing noncommuting Algebra. Available from http://math.ucsd.edu/ ncalg, 1996. Beware that to perform the computations in this paper you need NCGB (See [HS97]).

5. Kronewitter FD. Noncommutative computer algebra in linear algebra and control theory. Ph.D. Thesis, University of California: San Diego, 2000.

6. Pan ZG, Basar T. $\mathrm{H}_{\infty}$ optimal control for singularly perturbed systems 1 . Perfect state measurements. Automatica 1993; 29:401-423.

7. Pan $\mathrm{ZG}$, Basar $\mathrm{T}$. $\mathrm{H}_{\infty}$ optimal control for singularly perturbed systems 2 . Imperfect state measurements. IEEE Transactions on Automatic Control 1994; 39:280-299.

8. Helton JW, James MR, McEneaney WM. Nonlinear control: the joys of having an extra sensor. Proceedings of the 37th IEEE CDC, Tampa, FL, U.S.A., December 16-18, 1998; 4:3609-13.

9. Mora F. Groebner Bases for Non-commutative Polynomial Rings, Lecture Notes in Computer Science, Springer: Berlin, 1986; 229:353-362.

10. Helton JW, Stankus M. Computer assistance for discovering formulas in system engineering and operator theory. Journal of Functional Analysis 1999; 161:289-368.

11. Kwakernaak H, Sivan R. Linear Optimal Control Systems. Wiley Interscience: New York, 1972.

12. Cox D, Little J, O'Shea D. Ideals, Varieties, and Algorithms: An Introduction to Computational Algebraic Geometry and Commutative Algebra. Undergraduate Texts in Mathematics, Springer; Berlin, 1992.

13. Green EL, Heath LS, Keller BJ. Opal: A system for computing noncommutative Gröbner bases. In Comon $\mathrm{H}$, editor. Eighth International Conference on Rewriting Techniques and Applications (RTA-97), Lecture Notes in Computer Science, Vol. 1232, Springer: Berlin, 1997, p. 331-334. 